\title{
Search for extraterrestrial point sources of high energy neutrinos with AMANDA-II using data collected in 2000-2002
}

M. Ackermann ${ }^{4}$ J. Ahrens,${ }^{11}$ X. Bai, ${ }^{1}$ R. Bay, ${ }^{9}$ M. Bartelt, ${ }^{2}$ S. W. Barwick, ${ }^{10}$ T. Becka, ${ }^{11}$ K. H. Becker, ${ }^{2}$ J. K. Becker, ${ }^{20}$ E. Bernardini, ${ }^{4}$ D. Bertrand, ${ }^{3}$ D. J. Boersma ${ }^{4}$ S. Böser, ${ }^{4}$ O. Botner, ${ }^{17}$ A. Bouchta, ${ }^{17}$ O. Bouhali, ${ }^{3}$ J. Braun, ${ }^{15}$ C. Burgess,${ }^{18}$ T. Burgess, ${ }^{18}$ T. Castermans, ${ }^{13}$ D. Chirkin, ${ }^{9}$ B. Collin, ${ }^{8}$ J. Conrad, ${ }^{17}$ J. Cooley, ${ }^{15}$ D. F. Cowen, ${ }^{8}$ A. Davour, ${ }^{17}$ C. De Clercq, ${ }^{19}$ T. DeYoung, ${ }^{12}$ P. Desiati, ${ }^{15, *}$ P. Ekström, ${ }^{18}$ T. Feser, ${ }^{11}$ T. K. Gaisser, ${ }^{1}$ R. Ganugapati, ${ }^{15}$ H. Geenen, ${ }^{2}$ L. Gerhardt, ${ }^{10}$ A. Goldschmidt, ${ }^{7}$ A. Groß, ${ }^{20}$ A. Hallgren, ${ }^{17}$ F. Halzen, ${ }^{15}$ K. Hanson, ${ }^{15}$ D. Hardtke, ${ }^{9}$ R. Hardtke,${ }^{15}$ T. Harenberg, ${ }^{2}$ T. Hauschildt, ${ }^{4}$ K. Helbing, ${ }^{7}$ M. Hellwig, ${ }^{11}$ P. Herquet, ${ }^{13}$ G. C. Hill, ${ }^{15}$ J. Hodges, ${ }^{15}$ D. Hubert, ${ }^{19}$ B. Hughey, ${ }^{15}$ P. O. Hulth, ${ }^{18}$ K. Hultqvist, ${ }^{18}$ S. Hundertmark, ${ }^{18}$ J. Jacobsen, ${ }^{7}$ K. H. Kampert, ${ }^{2}$ A. Karle, ${ }^{15}$ J. L. Kelley, ${ }^{15}$ M. Kestel, ${ }^{8}$ G. Kohnen, ${ }^{13}$ L. Köpke, ${ }^{11}$ M. Kowalski, ${ }^{4}$ M. Krasberg, ${ }^{15}$ K. Kuehn, ${ }^{10}$ H. Leich, ${ }^{4}$ M. Leuthold, ${ }^{4}$ I. Liubarsky, ${ }^{5}$ J. Lundberg, ${ }^{17}$ J. Madsen, ${ }^{16}$ P. Marciniewski, ${ }^{17}$ H. S. Matis, ${ }^{7}$ C. P. McParland,${ }^{7}$ T. Messarius,${ }^{20}$ Y. Minaeva, ${ }^{18}$

P. Miočinović, ${ }^{9}$ R. Morse, ${ }^{15}$ K. Münich,${ }^{20}$ R. Nahnhauer, ${ }^{4}$ J. W. Nam, ${ }^{10}$ T. Neunhöffer, ${ }^{11}$ P. Niessen, ${ }^{1}$ D. R. Nygren, ${ }^{7}$ H. Ögelman, ${ }^{15}$ Ph. Olbrechts, ${ }^{19}$ C. Pérez de los Heros, ${ }^{17}$ A. C. Pohl,${ }^{6}$ R. Porrata, ${ }^{9}$ P. B. Price, ${ }^{9}$ G. T. Przybylski, ${ }^{7}$ K. Rawlins, ${ }^{15}$ E. Resconi, ${ }^{4}$ W. Rhode,${ }^{20}$ M. Ribordy,${ }^{13}$ S. Richter, ${ }^{15}$ J. Rodríguez Martino, ${ }^{18}$ H. G. Sander, ${ }^{11}$ K. Schinarakis, ${ }^{2}$ S. Schlenstedt, ${ }^{4}$ D. Schneider,${ }^{15}$ R. Schwarz, ${ }^{15}$ A. Silvestri, ${ }^{10}$ M. Solarz, ${ }^{9}$ G. M. Spiczak,${ }^{16}$ C. Spiering, ${ }^{4}$ M. Stamatikos, ${ }^{15}$ D. Steele, ${ }^{15}$ P. Steffen, ${ }^{4}$ R. G. Stokstad, ${ }^{7}$ K. H. Sulanke,,${ }^{4}$ I. Taboada,${ }^{14}$ O. Tarasova, ${ }^{4}$ L. Thollander, ${ }^{18}$ S. Tilav, ${ }^{1}$ W. Wagner, ${ }^{20}$ C. Walck, ${ }^{18}$ M. Walter, ${ }^{4}$ Y. R. Wang, ${ }^{15, \dagger}$ C. Wendt, ${ }^{15}$ C. H. Wiebusch, ${ }^{2}$ R. Wischnewski, ${ }^{4}$ H. Wissing, ${ }^{4}$ K. Woschnagg, ${ }^{9}$ and G. Yodh ${ }^{10}$

\author{
${ }^{1}$ Bartol Research Institute, University of Delaware, Newark, Delaware 19716, USA \\ ${ }^{2}$ Department of Physics, Bergische Universität Wuppertal, D-42097 Wuppertal, Germany \\ ${ }^{3}$ Université Libre de Bruxelles, Science Faculty CP230, B-1050 Brussels, Belgium \\ ${ }^{4}$ DESY, D-15735, Zeuthen, Germany \\ ${ }^{5}$ Blackett Laboratory, Imperial College, London SW7 2BW, United Kingdom \\ ${ }^{6}$ Department of Technology, Kalmar University, S-39182 Kalmar, Sweden \\ ${ }^{7}$ Lawrence Berkeley National Laboratory, Berkeley, California 94720, USA \\ ${ }^{8}$ Department of Physics, Pennsylvania State University, University Park, Pennsylvania 16802, USA \\ ${ }^{9}$ Department of Physics, University of California, Berkeley, California 94720, USA \\ ${ }^{10}$ Department of Physics and Astronomy, University of California, Irvine, California 92697, USA \\ ${ }^{11}$ Institute of Physics, University of Mainz, Staudinger Weg 7, D-55099 Mainz, Germany \\ ${ }^{12}$ Department of Physics, University of Maryland, College Park, Maryland 20742, USA \\ ${ }^{13}$ University of Mons-Hainaut, 7000 Mons, Belgium \\ ${ }^{14}$ Departamento de Física, Universidad Simón Bolivar, Caracas, 1080, Venezuela \\ ${ }^{15}$ Department of Physics, University of Wisconsin, Madison, Wisconsin 53706, USA \\ ${ }^{16}$ Physics Department, University of Wisconsin, River Falls, Wisconsin 54022, USA \\ ${ }^{17}$ Division of High Energy Physics, Uppsala University, S-75121 Uppsala, Sweden \\ ${ }^{18}$ Department of Physics, Stockholm University, SE-10691 Stockholm, Sweden \\ ${ }^{19}$ Vrije Universiteit Brussel, Dienst ELEM, B-1050 Brussels, Belgium \\ ${ }^{20}$ Institute of Physics, University of Dortmund, D-44221 Dortmund, Germany
}

(Received 13 December 2004; published 4 April 2005)

\begin{abstract}
The results of a search for point sources of high energy neutrinos in the northern hemisphere using data collected by AMANDA-II in the years 2000, 2001, and 2002 are presented. In particular, a comparison with the single-year result previously published shows that the sensitivity was improved by a factor of 2.2. The muon neutrino flux upper limits on selected candidate sources, corresponding to an $E_{\nu}^{-2}$ neutrino energy spectrum, are included. Sky grids were used to search for possible excesses above the background of cosmic ray induced atmospheric neutrinos. This search reveals no statistically significant excess for the three years considered.
\end{abstract}

DOI: $10.1103 /$ PhysRevD.71.077102

PACS numbers: 95.85.Ry, 95.55.Vj, 96.40.Tv, 98.54.-h

\section{INTRODUCTION}

*Corresponding author.

Email address: desiati@amanda.wisc.edu

${ }^{\dagger}$ Corresponding author.

Email address: yrwang@amanda.wisc.edu
In this communication, we update a previously published search for high energy neutrino point sources from the data collected by AMANDA-II [1] in 2000 [2], using the three-year sample from 2000 to 2002 . The sensitivity 
for the detection of point sources has constantly improved in AMANDA-II, starting from 1997 [3] and 1999 data [4], due to both detector performance and analysis technique improvements. The search for possible extraterrestrial high energy neutrinos assumes the signal has a $d N / d E_{\nu} \propto E_{\nu}^{-2}$ energy spectrum, as predicted by the Fermi acceleration mechanism. The atmospheric neutrinos which have a much steeper spectrum represent the background in which the signal is searched for.

This analysis, for most aspects similar to that presented and deeply discussed in [2], includes an effective requirement on the minimum energy of the reconstructed events which improves the sensitivity for high energy neutrino detection. Namely the sensitivity for this analysis is $\sim 2.2$ times better than previously obtained for the year 2000. This improvement is better than what would be expected from the longer exposure alone in the presence of background.

\section{DATA ANALYSIS}

The data used for this analysis were collected between the months of February and November in the years 2000, 2001 and 2002 (see Table I).

The experimental sample used in this analysis corresponds to a total of 607 days of live time and contains almost $5.6 \times 10^{9}$ triggers. Starting from 2002, a first level filter is performed at the south pole during data taking. The reduced amount of data is transferred via satellite to the northern hemisphere for analysis. After the application of an iterative maximum-likelihood reconstruction algorithm and the selection of tracks that are likely to be up going [5], about $0.45 \times 10^{6}$ events with reconstructed declination $\delta>-10^{\circ}$ remain. Since AMANDA-II is located at the south pole, $\delta=0^{\circ}$ corresponds to horizontal and $\delta=90^{\circ}$ to vertical up-going directions. These events, containing mostly the residual component of atmospheric muons and a contribution of atmospheric neutrinos, were used as the experimental background against which the signal selection is optimized.

To avoid biasing the event selection the data were scrambled by randomizing the reconstructed right ascension $(\alpha)$ of each event. The optimization procedure used in this analysis is similar to that described in [2], the differ-

TABLE I. The experimental live time and number of triggered events for each year used in this analysis. The triggered events may vary in different years mostly due to different cleaning procedures, which are mainly affected by the number of stable optical modules during the specific year.

\begin{tabular}{lcc}
\hline \hline Year & Live time (days) & Triggers \\
\hline 2000 & 197 & $1.34 \times 10^{9}$ \\
2001 & 194 & $2.04 \times 10^{9}$ \\
2002 & 216 & $2.17 \times 10^{9}$ \\
\hline \hline
\end{tabular}

ence being in the choice of observables. Instead of a neural network parameter the number of hit optical modules for each event (number of channels or nch) is used, along with the reconstructed track length in the array and the likelihood ratio between the muon track reconstruction and a muon reconstruction constrained by using an atmospheric muon prior [6]. A full simulation chain, including neutrino absorption in the Earth, neutral current regeneration, muon propagation and detector response for the given data taking periods, is used to simulate point sources of muon neutrinos and antineutrinos [2]. Events are simulated at the center of each $5^{\circ}$ band of declination $(\delta)$, according to an $E_{\nu}^{-2}$ energy spectrum. The final cuts on these observables and the optimum size of each circular search bin were independently determined for each declination band in order to have the strongest constraint on the signal hypothesis. This corresponds to the best sensitivity, i.e. the average flux upper limit obtained in an ensemble of identical experiments assuming no signal [7]. The resulting zenith-dependent median pointing resolution varies between $1.5^{\circ}$ and $2.5^{\circ}$. The true directional information was then restored for the calculation of the limits.

The upper limits of this analysis were calculated using the background $n_{b}$ measured using the events off source in the corresponding declination band, and the expected number of events, $n_{s}$, from a simulated point source of known flux $\Phi\left(E_{\nu}\right): \Phi_{\text {limit }}\left(E_{\nu}\right)=\Phi\left(E_{\nu}\right) \times \mu_{90}\left(n_{\text {obs }}, n_{b}\right) / n_{s}$. Here $n_{\text {obs }}$ is the number of observed events in the given source bin, and $\mu_{90}$ is the upper limit on the number of events following the unified ordering prescription of Feldman and Cousins [8]. The three years were analyzed both separately and as combined data samples.

The absolute normalization of the atmospheric neutrino simulation (with the flux from [9]) with respect to the selected experimental events was determined to be $1.03 \pm$ 0.02 (statistical error only). The used flux was compared with several more recent calculations and confirmed that it gives a reasonable representation of the neutrino intensity over the range of energies and angles relevant for AMANDA-II. The response of AMANDA-II is such that most of the atmospheric neutrino signal comes from muon neutrinos and antineutrinos with energies between $\sim 50 \mathrm{GeV}$ and $\sim 100 \mathrm{TeV}[10,11]$. The intrinsic theoretical uncertainty of the atmospheric neutrino flux in this energy region was taken to be about $30 \%[12,13]$, even if above $10 \mathrm{TeV}$ it could be significantly higher. The overall experimental systematic uncertainty in the acceptance was evaluated using the down-going muon flux, and it is $\sim 30 \%$ [14]. This includes the detector efficiency and the optical properties of the fiducial ice, needed for the detector simulation, which were determined using down-going muon data and in situ calibration lasers [15]. The absolute pointing accuracy, determined with coincident events between the SPASE air shower array [16] and AMANDA-II, is better than $1^{\circ}$, i.e. smaller than the angular resolution. 
These systematic uncertainties do not affect significantly the limit calculations [2].

\section{RESULTS}

Figure 1 shows the calculated sensitivity versus declination for energies above $10 \mathrm{GeV}$. The event selection used produces a sensitivity which is fairly constant over all declinations. For $0^{\circ}<\delta<5^{\circ}[0<\sin (\delta)<0.09]$ the background contamination is 4 times higher than for $\delta>$ $5^{\circ}$, and the sensitivity is poorer. For $\delta>80^{\circ}[\sin (\delta)>$ $0.98]$, on the other hand, the solid angle aperture is small and the background evaluation is affected by higher relative statistical fluctuations.

The final three-year sample consists of 646 upward $(\delta>$ $5^{\circ}$ ) reconstructed muons (see Table II). The predicted number of atmospheric neutrinos is 635. In the year 2000 alone, the number of selected events is 306 , compared with the 601 (699 for $\delta>0^{\circ}$ ) in Ref. [2]. The difference between the two samples is due to the different choice of observables used for the selection optimization. In particular, the use of the nch observable, which is correlated to the energy released by the muon in the array and, ultimately, to the neutrino energy, selects $\sim 26 \%$ higher median energies than those in [2] (from $\sim 700 \mathrm{GeV}$ to $\sim 1 \mathrm{TeV}$ for a single year). This selection is obtained at the price of removing a significant fraction of atmospheric neutrino events: for instance only 221 events in [2] would survive the new selection, 94\% of which (i.e. 207) are also found in the new sample from the year 2000 .

As shown in Table II the number of events in the final sample $\left(n_{\text {obs }}\right)$ is not the sum of the ones selected in the individual years, because the event selection has been reoptimized with the 3 times larger exposure. The energy cut becomes more stringent with increasing exposure and

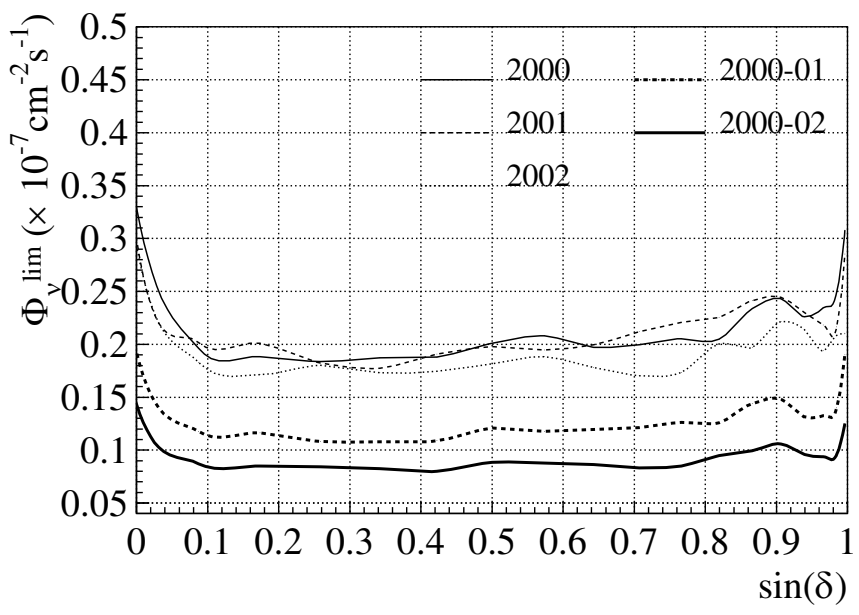

FIG. 1. Sensitivities on the integrated flux above $E_{\nu}=$ $10 \mathrm{GeV}$ as a function of declination and for an $E_{\nu}^{-2}$ energy spectrum. The sensitivities for the years 2000, 2001 and 2002 are compatible with each other, and shown along with the one for 2000-2001 and for the 2000-2002 three-year sample.
TABLE II. The number of observed events with $\delta>5^{\circ}$ after cut optimization, for each year and the combined three-year sample. The numbers relative to Ref. [2] are compatible with a normalization factor of $\sim 0.86$, for the atmospheric neutrino simulation, as quoted in the above reference. The numbers $n_{p}$ of the predicted atmospheric and signal neutrino events (with signal energy spectrum of $d \Phi_{\nu_{\mu}} / d E_{\nu}=10^{-6} \times$ $E_{\nu}^{-2} \mathrm{~cm}^{-2} \mathrm{~s}^{-1} \mathrm{sr}^{-1} \mathrm{GeV}^{-1}$ ) are also shown.

\begin{tabular}{lccc}
\hline \hline Year & $n_{\mathrm{obs}}$ & $n_{p}\left(\nu_{\mu}^{\mathrm{atm}}\right)$ & $n_{p}\left(\nu_{\mu}^{\mathrm{sig}}\right)$ \\
\hline $2000[2]$ & 601 & 676 & 133 \\
2000 & 306 & 296 & 111 \\
2001 & 347 & 364 & 115 \\
2002 & 429 & 429 & 131 \\
$2000-2002$ & 646 & 635 & 297 \\
\hline \hline
\end{tabular}

the median energy of the three-year selected sample is $\sim 1.3 \mathrm{TeV}$ (and extending up to $\sim 100 \mathrm{TeV}$ ). Consequently the three-year sample contains $\sim 40 \%$ fewer observed events than the sum of single years, but only $\sim 17 \%$ of the high energy neutrino signal events are lost.

The detector performance is assessed by the neutrino effective area $A_{\text {eff }}^{\nu}\left(E_{\nu}, \delta\right)$, which contains the neutrino interaction probability, muon propagation, detector response and the analysis selection. It is defined by the relation between the differential neutrino flux $d \Phi_{\nu} / d \Omega d E_{\nu}$ and the predicted number of neutrino events $n_{p}(\nu)$, through the equation

$$
n_{p}(\nu)=T_{\text {live }} \cdot \int_{\Omega} \int_{E_{\nu}^{\min }}^{E_{\text {max }}^{\max }} A_{\mathrm{eff}}^{\nu}\left(E_{\nu}, \delta\right) \frac{d \Phi_{\nu}}{d \Omega d E_{\nu}} d \Omega d E_{\nu}
$$

Figure 2 shows the muon neutrino effective area as a function of neutrino energy for the three-year optimized selection. The curves are shown for different declinations.

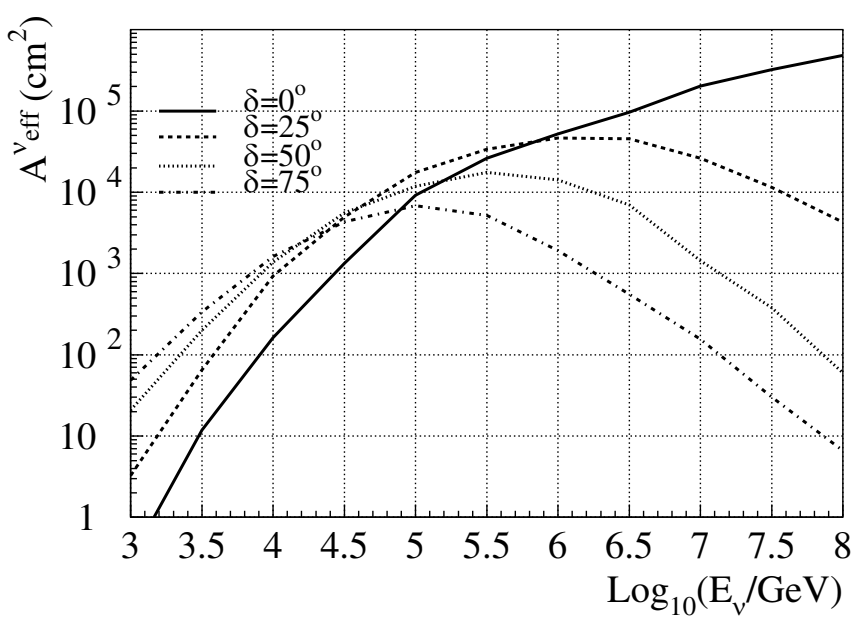

FIG. 2. Muon neutrino effective area as a function of the neutrino energy at different declinations. The effect of neutrino absorption in the Earth is responsible for the effective area decrease at high energies and declinations. 
Above $10^{6} \mathrm{GeV}$ neutrinos begin to be absorbed by the Earth, except for the events that enter AMANDA-II horizontally. As shown in Table II we expect the present effective area to be smaller than in [2], nevertheless its overall reduction is more enhanced at low energies, where the signal is not expected to be particularly significant.

A binned search for excesses in the $5^{\circ}<\delta<85^{\circ}$ region was performed on the three-year event sample. The search grid contains 290 rectangular bins with declinationdependent width ranging from $5.6^{\circ}$ to $8.8^{\circ}$, based on the optimized search bin diameter. The grid is shifted 4 times in $\delta$ and $\alpha$ to fully cover boundaries between the bins of the original configuration. A higher number of grid shifts showed no improvement in the average maximum statisti-

TABLE III. 90\% C.L. upper limits on candidate sources. Results from the present analysis are reported for a comparison with the limits from [2]. Limits are for the assumed $E_{\nu}^{-2}$ spectral shape, integrated above $E_{\nu}=10 \mathrm{GeV}$, and in units of $10^{-8} \mathrm{~cm}^{-2} \mathrm{~s}^{-1}\left(\Phi_{\nu}^{\lim }\right)$

\begin{tabular}{lrrrrrrrr}
\hline \hline & \multicolumn{7}{c}{ From [2] } & \multicolumn{5}{c}{ This work } \\
Candidate & $\delta\left(^{\circ}\right)$ & $\alpha(h)$ & $n_{\text {obs }}$ & $n_{b}$ & $\Phi_{\nu}^{\text {lim }}$ & $n_{\text {obs }}$ & $n_{b}$ & $\Phi_{\nu}^{\text {lim }}$ \\
\hline TeV Blazars & & & & & & & & \\
Markarian 421 & 38.2 & 11.07 & 3 & 1.50 & 3.5 & 0 & 1.35 & 0.34 \\
Markarian 501 & 39.8 & 16.90 & 1 & 1.57 & 1.8 & 3 & 1.31 & 1.49 \\
1ES 1426+428 & 42.7 & 14.48 & 1 & 1.62 & 1.7 & 2 & 1.13 & 1.16 \\
1ES 2344+514 & 51.7 & 23.78 & 1 & 1.23 & 2.0 & 1 & 1.25 & 0.82 \\
1ES 1959+650 & 65.1 & 20.00 & 0 & 0.93 & 1.3 & 0 & 1.59 & 0.38 \\
GeV Blazars & & & & & & & & \\
QSO 0528+134 & 13.4 & 5.52 & 1 & 1.09 & 2.0 & 1 & 1.88 & 0.57 \\
QSO 0235+164 & 16.6 & 2.62 & 1 & 1.49 & 1.7 & 3 & 2.15 & 1.12 \\
QSO 1611+343 & 34.4 & 16.24 & 0 & 1.29 & 0.8 & 0 & 1.66 & 0.31 \\
QSO 1633+382 & 38.2 & 16.59 & 1 & 1.50 & 1.7 & 1 & 1.33 & 0.75 \\
QSO 0219+428 & 42.9 & 2.38 & 1 & 1.63 & 1.6 & 0 & 1.15 & 0.37 \\
QSO 0954+556 & 55.0 & 9.87 & 1 & 1.66 & 1.7 & 2 & 1.04 & 1.50 \\
QSO 0716+714 & 71.3 & 7.36 & 2 & 0.74 & 4.4 & 3 & 0.93 & 1.91 \\
Microquasars & & & & & & & & \\
SS433 & 5.0 & 19.20 & 0 & 2.38 & 0.7 & 1 & 2.21 & 0.55 \\
GRS 1915+105 & 10.9 & 19.25 & 1 & 0.91 & 2.2 & 3 & 1.84 & 1.26 \\
GRO J0422+32 & 32.9 & 4.36 & 2 & 1.31 & 2.9 & 2 & 1.49 & 1.08 \\
Cygnus X1 & 35.2 & 19.97 & 2 & 1.34 & 2.5 & 0 & 1.59 & 0.31 \\
Cygnus X3 & 41.0 & 20.54 & 3 & 1.69 & 3.5 & 1 & 1.26 & 0.75 \\
XTE J1118+480 & 48.0 & 11.30 & 1 & 0.92 & 2.2 & 1 & 1.12 & 0.80 \\
CI Cam & 56.0 & 4.33 & 0 & 1.72 & 0.8 & 2 & 1.05 & 1.44 \\
LS I +61 303 & 61.2 & 2.68 & 0 & 0.75 & 1.5 & 5 & 1.67 & 2.43 \\
SNR, magnetars and miscellaneous & & & & & \\
SGR 1900+14 & 9.3 & 19.12 & 0 & 0.97 & 1.0 & 2 & 1.78 & 0.94 \\
Crab Nebula & 22.0 & 5.58 & 2 & 1.76 & 2.4 & 4 & 1.86 & 1.43 \\
Cassiopeia A & 58.8 & 23.39 & 0 & 1.01 & 1.2 & 2 & 1.12 & 1.38 \\
3EG J0450+1105 & 11.4 & 4.82 & 2 & 0.89 & 3.2 & 1 & 1.83 & 0.59 \\
M 87 & 12.4 & 12.51 & 0 & 0.95 & 1.0 & 3 & 1.83 & 1.24 \\
Geminga & 17.9 & 6.57 & 3 & 1.78 & 3.3 & 2 & 2.06 & 0.81 \\
UHE CR Triplet & 20.4 & 1.28 & 2 & 1.84 & 2.3 & 0 & 2.15 & 0.20 \\
NGC 1275 & 41.5 & 3.33 & 1 & 1.72 & 1.6 & 1 & 1.14 & 0.78 \\
Cyg. OB2 region. & 41.5 & 20.54 & 3 & 1.72 & 3.5 & 1 & 1.14 & 0.78 \\
\hline \hline & & & & & & & &
\end{tabular}

cal significances on the simulated Poisson-fluctuated signal with intensities comparable to the background. The probability distribution for background fluctuations in the ensemble of bins was evaluated by using 20000 experimental samples with scrambled $\alpha$ and calculating the highest value of the maximum statistical fluctuation significance over the entire sky.

The bin with the most statistically significant excess from the three-year experimental sample is at about $\alpha=$ $22 \mathrm{~h}$ and $\delta=21^{\circ}$, with 10 observed events in the search bin on a background of 2.38 events, estimated from the corresponding declination band. The observed excess has a statistical significance of $1.9 \times 10^{-4}(3.73 \sigma)$. The chance probability of such an excess, in the ensemble of bins, is $28 \%$.

Table III shows the $90 \%$ C.L. neutrino flux limits for northern hemisphere $\mathrm{TeV}$ blazars, selected $\mathrm{GeV}$ blazars, microquasars, magnetars and selected miscellaneous candidates. The limits are compared with the values from [2]; they are compatible with the average flux upper limit, or sensitivity, of Fig. 1 and the deviations from it are due to statistical fluctuations in the observed sample.

Figure 3 shows the $90 \%$ C.L. neutrino flux upper limits in equatorial coordinates. The limits are calculated by scanning the sky and counting the events within the optimized search bins at the given declination. The highest upper limit in the figure corresponds to the previously discussed statistically significant bin. Other high limit spots visible in the figure have statistical significances smaller than $3.4 \sigma$.

We analyzed the 2000-2002 data sample collected by the AMANDA-II detector to search for point sources of high energy neutrinos. We performed both a nontargeted

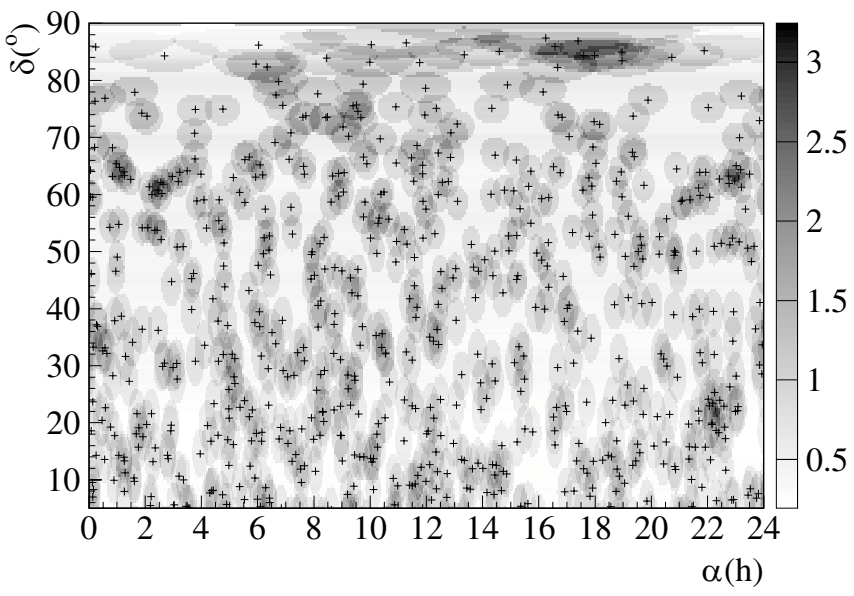

FIG. 3. 2000-2002 upper limits (90\% C.L.) on the neutrino flux integrated above $10 \mathrm{GeV}$ in equatorial coordinates for $\delta>$ $5^{\circ}$. Limits (scale on right axis) are given in units of $\times 10^{-8} \mathrm{~cm}^{-2} \mathrm{~s}^{-1}$ for the assumed $E^{-2}$ spectrum. Systematic uncertainties are not included. The cross symbols represent the observed events. 
binned search and a targeted search focusing on known objects that are potential high energy neutrino emitters (as in Ref. [2]). The sensitivity on the neutrino flux integrated above $E_{\nu}=10 \mathrm{GeV}$ is $\sim 9 \times 10^{-9} \mathrm{~cm}^{-2} \mathrm{~s}^{-1}$. We found no evidence of a significant flux excess above the background. A km-scale experiment, such as IceCube [17], will be able to increase the detection sensitivity by at least a factor of 30 in the same time scale above $1 \mathrm{TeV}$.

\section{ACKNOWLEDGMENTS}

We acknowledge the support of the following agencies: National Science Foundation-Office of Polar Programs, National Science Foundation-Physics Division, University of Wisconsin Alumni Research Foundation, Department of
Energy, and National Energy Research Scientific Computing Center (supported by the Office of Energy Research of the Department of Energy), UC-Irvine AENEAS Supercomputer Facility, USA; Swedish Research Council, Swedish Polar Research Secretariat, and Knut and Alice Wallenberg Foundation, Sweden; German Ministry for Education and Research, Deutsche Forschungsgemeinschaft (DFG), Germany; Fund for Scientific Research (FNRS-FWO), Flanders Institute to encourage scientific and technological research in industry (IWT), and Belgian Federal Office for Scientific, Technical and Cultural affairs (OSTC), Belgium; I. T. acknowledges support from Fundación Venezolana de Promoción al Investigador (FVPI), Venezuela; D. F. C. acknowledges the support of the NSF CAREER program.
[1] E. Andrés et al., Astropart. Phys. 13, 1 (2000).

[2] J. Ahrens et al., Phys. Rev. Lett. 92, 071102 (2004).

[3] J. Ahrens et al., Astrophys. J. 583, 1040 (2003).

[4] P. Desiati et al., in Proceedings of the 19th European Cosmic Ray Symposium, Florence, Italy, 2004 [Int. J. Mod. Phys. A (to be published)].

[5] J. Ahrens et al., Nucl. Instrum. Methods Phys. Res., Sect. A 524, 169 (2004).

[6] G. C. Hill, in Proceedings of the 27th ICRC, Hamburg, Germany, 2001, edited by K.-H. Kampert, G. Hainzelmann, and C. Spiering (Copernicus Gesellschaft e.V., Katlemburg-Lindau, Germany, 2001), Vol. HE 267, p. 1279.

[7] G. C. Hill and K. Rawlins, Astropart. Phys. 19, 393 (2003).

[8] G. J. Feldman and R. D. Cousins, Phys. Rev. D 57, 3873 (1998).

[9] P. Lipari, Astropart. Phys. 1, 195 (1993).
[10] E. Andrés et al., Nature (London) 410, 441 (2001)

[11] J. Ahrens et al., Phys. Rev. D 66, 012005 (2002).

[12] T. K. Gaisser and M. Honda, Annu. Rev. Nucl. Part. Sci. 52, 153 (2002).

[13] M. Honda et al., Phys. Rev. D 70, 043008 (2004).

[14] P. Desiati et al., in Proceedings of the 28th ICRC, Tsukuba, Japan, edited by T. Kajita, Y. Asaoka, A. Kawachi, Y. Matsubara, and M. Sasaki (Universal Academy Press, Inc., Tokyo, 2003), Vol. HE 2.3, p. 1373.

[15] K. Woschnagg et al., in Proceedings of the 21st International Conference On Neutrino Physics And Astrophysics (Neutrino 2004), Paris, France, 2004 [Nucl. Phys. B, Proc. Suppl. (to be published)], astro-ph/ 0409423.

[16] J. E. Dickinson et al., Nucl. Instrum. Methods Phys. Res., Sect. A 440, 95 (2000).

[17] J. Ahrens et al., Astropart. Phys. 20, 507 (2004). 